\title{
Increased Frontal Gamma and Posterior Delta Powers as Potential Neurophysiological Correlates Differentiating Posttraumatic Stress Disorder from Anxiety Disorders
}

\author{
Sun-Young Moon ${ }^{1}$, Yoo Bin Choi', Hee Kyung Jung ${ }^{1}$, Yoonji Irene Lee', and Soo-Hee Choi ${ }^{1,2} \bowtie$ \\ ${ }^{1}$ Department of Psychiatry, Seoul National University Hospital, Seoul, Republic of Korea \\ ${ }^{2}$ Department of Psychiatry and Institute of Human Behavioral Medicine, Seoul National University College of Medicine, Seoul, Republic of Korea
}

\begin{abstract}
Objective Posttraumatic stress disorder (PTSD) is distinct from anxiety disorders in its etiology and clinical symptomatology, and was reclassified into trauma- and stressor-related disorders in DSM-5. This study aimed to find neurophysiological correlates differentiating PTSD from anxiety disorders using resting-state quantitative electroencephalography (qEEG).

Methods Thirty-six patients with either PTSD or acute stress disorder and 79 patients with anxiety disorder were included in the analysis. qEEG data of absolute and relative powers and patients' medication status on the day of qEEG examination were obtained. Electrodes were grouped into frontal, central, and posterior regions to analyze for regional differences. General linear models were utilized to test for group differences in absolute and relative powers while controlling for medications.

Results PTSD patients differed from those with anxiety disorders in overall absolute powers $[F(5,327)=2.601, p=0.025]$. Specifically, overall absolute delta powers $[\mathrm{F}(1,331)=4.363, \mathrm{p}=0.037]$, and overall relative gamma powers $[\mathrm{F}(1,331)=3.965$, $\mathrm{p}=0.047]$ were increased in PTSD group compared to anxiety disorder group. Post hoc analysis regarding brain regions showed that the increase in absolute delta powers were localized to the posterior region $[\mathrm{F}(1,107)=4.001, \mathrm{p}=0.048]$. Additionally, frontal absolute gamma powers $[\mathrm{F}(1,107)=4.138$, $\mathrm{p}=0.044]$ were increased in PTSD group compared to anxiety disorder group.

Conclusion Our study suggests increased overall absolute delta powers and relative gamma powers as potential markers that could differentiate PTSD from anxiety disorders. Moreover, increased frontal absolute gamma and posterior delta powers might pose as novel markers of PTSD, which may reflect its distinct symptomatology.

Psychiatry Investig 2018;15(11):1087-1093
\end{abstract}

Key Words Posttraumatic stress disorder, Anxiety disorders, Quantitative electroencephalography.

\section{INTRODUCTION}

Posttraumatic stress disorder (PTSD) and anxiety disorders share a common symptomatology of abnormal and elevated fear response, ${ }^{1}$ which is thought to underlie heightened amygdala and insula activities. ${ }^{2}$ For their common symptoms and neurobiology, PTSD was classified as an anxiety disorder until Diagnostic and Statistical Manual of mental disorders, fourth edition (DSM-IV). ${ }^{3}$

Received: July 6, 2018 Revised: July 17, 2018

Accepted: September 30, 2018

$\triangle$ Correspondence: Soo-Hee Choi, $\mathrm{MD}, \mathrm{PhD}$

Department of Psychiatry, Seoul National University Hospital, 101 Daehakro, Jongno-gu, Seoul 03080, Republic of Korea

Tel: +82-2-2072-3173, Fax: +82-2-744-7241, E-mail: soohchoi@snu.ac.kr

(a) This is an Open Access article distributed under the terms of the Creative Commons Attribution Non-Commercial License (http://creativecommons.org/licenses/by$\mathrm{nc} / 4.0$ ) which permits unrestricted non-commercial use, distribution, and reproduction in any medium, provided the original work is properly cited.
However, due to its etiology of explicit trauma and the distinct symptomatology which includes re-experiencing phenomena, avoidance, and alterations in $\operatorname{mood} / \operatorname{cog}$ nition, PTSD was separated from anxiety disorders and newly classified as trauma- and stressor-related disorders in DSM-5. ${ }^{4}$ Recent neurobiological findings are also in support of this reclassification as, although hyperactivation of the amygdala and insula is a common feature found in both PTSD and anxiety disorders, ${ }^{2}$ only PTSD is associated with hypoactivation of the ventromedial prefrontal cortex (vmPFC). ${ }^{5}$ The vmPFC is known to have direct inhibitory connections with the amygdala, and has been hypothesized to play a role in the extinction of conditioned fear responses, ${ }^{6,7}$ and also to regulate arousal under stress and promote resilience. $.^{7-12} \mathrm{De}-$ creased vmPFC function in PTSD are associated with more grave symptom severity which could contribute to the dysregulation of mood observed in this disorder. ${ }^{13-15}$ 
The electroencephalography (EEG) findings of anxiety disorders have largely varied, and the majority of studies have investigated a single disorder of the many anxiety disorders. Among them, many studies have focused on predicting the outcome of cognitive-behavioral therapy, and some have shown that greater frontal asymmetry was associated with poorer treatment outcomes. ${ }^{16}$ One study comparing the quantitative EEG (qEEG) data of individuals with panic disorder with those of healthy controls found that overall delta, theta, and alpha absolute powers were increased in panic disorder and that the absolute delta and alpha powers significantly correlated with observer ratings of anxiety. ${ }^{17}$ Among individuals with social phobia, qEEG findings showed decreases in the absolute and relative delta and theta powers, and that trait anxiety and depression negatively correlated with absolute theta powers compared to healthy controls. ${ }^{18}$

There have been even fewer studies with more varying results on the EEG findings in PTSD. One study reported veterans with PTSD had increased theta powers, ${ }^{19}$ while others did not. ${ }^{20,21}$ Another study reported that PTSD veterans in comparison to veterans without symptoms had decreased alpha and increased beta powers. ${ }^{22}$ One study evaluating functional connectivity of resting-state EEG in PTSD patients reported results of reduced connection strength and communication efficiency in beta and gamma frequency bands, ${ }^{23}$ and another study reported disrupted global indices (strength, clustering coefficient, path length, and efficiency) in delta, theta, low beta frequency bands of resting-state EEG in PTSD subjects. ${ }^{24}$ A more recent study of bereaved families following a national trauma has reported increased frontal beta powers, suggesting possible compensatory mechanisms following a complex trauma. ${ }^{25}$

However, it is difficult to explain the results of previous EEG studies in relation to the pathophysiology of PTSD and anxiety disorders due to the inconsistent and divergent nature of study designs and results. EEG studies of anxiety disorders were likely to focus on EEG findings that could predict treatment response to cognitive-behavioral therapy rather than on the inherent EEG features that could explain the neurobiology of the disorder. In the case of PTSD, there have been even fewer EEG studies, and the results varied widely among them, making it hard to allow for a consistent interpretation. Also, to our knowledge, there have been no previous studies directly comparing PTSD with anxiety disorders. Furthermore, there was no EEG study of PTSD controlling for the effect of psychotropic medications, and the interpretation of results of the studies with drug-free participants was limited with the problem of a deficient number of study participants.

Therefore, the present study was performed to identify the neurobiological features capable of differentiating PTSD from anxiety disorders using qEEG. We hypothesized that the powers of EEG frequency bands would differ between the two disorder categories and that they may reflect different symptomatology. As medications could modify EEG findings, we quantified and standardized medications into equivalent doses of specific categories of drugs to control for them when testing differences of absolute and relative powers between the two study groups.

\section{METHODS}

\section{Participants}

Among the patients examined by qEEG in the Psychiatry Department of Seoul National University Hospital between 2010 and 2016, data from those between the ages of 20 and 55 years old with a primary diagnosis of either PTSD or acute stress disorder (ASD; $\mathrm{n}=72$ ) and those with a primary diagnosis of anxiety disorder $(n=133)$ were initially acquired through a retrospective review of the medical records. The diagnosis was made by at least one psychiatrist with a board certificate, according to the diagnostic criteria of the Classification of Mental and Behavioral Disorders by the 10th revision of the International Classification of Diseases (ICD-10) ${ }^{26}$

The exclusion criteria were as followed: 1) a comorbid diagnosis of either psychotic or substance use disorder or intellectual disability; 2) drowsiness or sleeping during the eyes-closed qEEG protocol (to exclude the effects of somnolence state on EEG findings); 3) extreme outliers of EEG powers during exploratory data analyses; 4) being treated with tricyclic antidepressants, opioid agents, anticonvulsant agents, lithium, psychostimulants, or anticholinergic agents (to control for the effects of medication); and 5) being treated with antipsychotic agents, benzodiazepine, or antidepressants at doses exceeding 2 standard deviations (i.e., those being treated with $\geq 5.28 \mathrm{mg}$ of an olanzapine-equivalent dose, $\geq 3.42 \mathrm{mg}$ of a lorazepam-equivalent dose, or $\geq 71.87 \mathrm{mg}$ of an imipramine-equivalent dose).

Thirty-six patients with PTSD $(n=32)$ or ASD $(n=4)$ and 79 patients with anxiety disorder were included in the final analysis. The anxiety disorder group included 36 patients with a diagnosis of panic disorder, 5 with social anxiety disorder, and 38 with generalized anxiety disorder or unspecified anxiety disorder. This study adhered to the tenets of the Declaration of Helsinki and the ICH-GCP revised in 2013. The research protocol of this study was approved by the Institutional Review Board at Seoul National University Hospital (Number: 1712-045-905).

\section{Standardization of medications}

Information regarding all neuropsychiatric drugs administered on the day of qEEG acquisition were collected. To allow for quantitative analysis, antipsychotic drugs were converted 
into olanzapine-equivalent doses, ${ }^{27}$ benzodiazepines into lorazepam-equivalent doses, ${ }^{28}$ and antidepressants into imipramine-equivalent doses; $150 \mathrm{mg}$ of imipramine was calculated as equivalent to $150 \mathrm{mg}$ of clomipramine, $30 \mathrm{mg}$ of fluoxetine, $45 \mathrm{mg}$ of citalopram, $150 \mathrm{mg}$ of fluvoxamine, $150 \mathrm{mg}$ of milnacipran, $125 \mathrm{mg}$ of sertraline, $30 \mathrm{mg}$ of paroxetine, $150 \mathrm{mg}$ of venlafaxine, $250 \mathrm{mg}$ of bupropion, according to previous studies. ${ }^{29}$ Doses of escitalopram and duloxetine were standardized as escitalopram doses, with $10 \mathrm{mg}$ of escitalopram corresponding to $60 \mathrm{mg}$ of duloxetine. ${ }^{30}$ Mirtazapine doses were independently quantified as there were no consensus concerning dose equivalency between mirtazapine and other antidepressant agents. After the standardization procedure, only a few non-standardized drugs remained, which were administered just in small doses: tianeptine ( $\mathrm{n}=26,12.5 \mathrm{mg}$ ), buspirone $(\mathrm{n}=14,5-10 \mathrm{mg})$, propranolol $(\mathrm{n}=18,10-40 \mathrm{mg})$, and trazodone $(\mathrm{n}=2,25 \mathrm{mg})$.

\section{EEG recording \& processing procedures}

All EEGs were measured and recorded by a single skilled engineer with SynAmps2 (Compumedics, Abbotsford, Victoria, Australia) and Neuroscan system (Scan 4.3; Compumedics) installed in the EEG room of Seoul National University Hospital. Patients were seated in a reclining chair in an isolated, shielded EEG room, and their neurophysiological signals were measured for about 20 minutes in a stable state with eyes closed. ${ }^{31}$ Twenty-one electrodes were attached to the scalp (Cz, FPz, Fz, Oz, Pz, T4, O2, T6, C4, P4, Fp2, F8, F4, T3, O1, T5, C3, P3, Fp1, F7, F3) according to the international 10-20 system, with two electrodes to track eye movements. Impedance was set at $<5 \mathrm{k} \Omega$, and reference nodes were attached to both mastoid processes for bipolar analyses. Data were collected at a frequency of $500 \mathrm{~Hz}$ and were band-pass filtered between 0.1 and $60 \mathrm{~Hz}$. For spectral analyses, data from the Neuroscan system were transferred to NeuroGuide software (NF 2.7.8; Applied Neuroscience, St. Petersburg, FL, USA) in a 32-bit file format. Stable epochs of approximately $90 \mathrm{sec}$ onds without muscle and ocular movement artifacts were selected manually by careful visual inspection of a psychiatrist, and a psychiatric specialist supervised this procedure. The objective value of split-half reliability and test re-test reliability was set at 0.95 for quality control, and those less than 0.90 were considered inadequate for analysis.

Selected epochs underwent Fast Fourier Transformations, and the absolute $(\mu \mathrm{V})$ powers for the delta $(0.5-4 \mathrm{~Hz})$, theta (4-8 Hz), alpha $(8-12 \mathrm{~Hz})$, beta $(12-30 \mathrm{~Hz})$, and gamma (30-40 Hz) frequency bands of each of 21 electrodes were acquired. Relative powers (\%) of each frequency band for each of the 21 electrodes were subsequently calculated.

Electrodes were then grouped into three regions according to previously reported studies, ${ }^{32}$ and arithmetic mean values of absolute and relative powers for each frequency bands (i.e., delta, theta, alpha, beta, and gamma) were obtained from each group of electrodes: frontal (FP1, F3, F7, Fz, Fp2, F4, $\mathrm{F} 8$ ), central (T3, C3, Cz, T4, C4), and posterior regions (T5, P3, O1, Pz, T6, P4, O2).

\section{Statistical analysis}

IBM SPSS Statistics version 19 (IBM Corp., Armonk, NY, USA) was used for statistical analysis. Differences between groups in demographic and clinical variables were tested using the t-test for continuous variables and the chi-square test for categorical variables.

General linear models were used to compare the overall absolute and relative powers between study groups. Study group (PTSD vs. anxiety disorders) and grouped regions (frontal, central, posterior) were entered as fixed factors. Absolute and relative powers were entered as independent variables. Doses of antipsychotic drugs, benzodiazepines, and antidepressants converted into equivalent doses of each drug categories and were entered as covariates. The results permitted analysis of the interaction effects by study group or region. There were no significant interaction effects of the region by the group, and we were able to test for the main effect of group on each frequency band in terms of the overall absolute or relative powers, except for overall absolute gamma powers. When significant differences were observed in specific frequency bands, we performed post hoc analysis to identify the grouped regions in which such differences were most pronounced and significant. Post hoc analysis was also performed using general linear models (medication dosages entered as covariates). And because we expected that there would be region by group interaction effects in the overall absolute powers of the gamma frequency band from the exploratory data analysis, we performed an additional post hoc analysis to evaluate whether potentially significant differences in the frontal grouped region were obscured by such interaction effects.

\section{RESULTS}

\section{Demographic and clinical characteristics}

The demographic and clinical characteristics of the 36 patients with PTSD or ASD and the 79 patients with anxiety disorder are presented in Table 1. There were no differences in age, gender, or handedness between the two groups.

The two groups did not differ with regard to benzodiazepine, antidepressant, or propranolol treatment (Table 2). However, those with PTSD were more likely to be treated with antipsychotic agents. Patients with PTSD were being treated with higher doses of antipsychotics and lower doses of antidepressants. 


\section{EEG activity: absolute powers}

After controlling for medication dose as a covariate, we found that the overall absolute power properties of the PTSD and anxiety disorder groups differed significantly $[F(5,327)=2.601$, $\mathrm{p}=0.025$ ] (Figure 1). Among the specific frequency bands, the overall absolute delta powers were significantly increased in the PTSD group $[\mathrm{F}(1,331)=4.363, \mathrm{p}=0.037]$. Post hoc analysis showed that absolute delta powers of the posterior cerebral region were significantly increased in the PTSD group compared to the anxiety-disorder group $[\mathrm{F}(1,107)=4.001, \mathrm{p}=0.048]$.

The overall absolute powers of other frequency bands did not differ between two groups. However, as we suspected region by group interaction effects in the gamma frequency band from the exploratory data analysis, we performed an additional post hoc analysis on the gamma frequency band. Result showed that absolute gamma powers of the frontal region were increased in PTSD compared to anxiety disorders $[F(1,107)=4.138, p=0.044]$.

\section{EEG activity: relative powers}

After controlling for medications, we found that the overall relative powers of the PTSD group did not differ from those of the anxiety disorder group. Among the specific frequency bands, the relative powers of the gamma band were significantly increased in the PTSD group $[\mathrm{F}(1,331)=3.965, \mathrm{p}=0.047]$ compared to the anxiety disorder group (Figure 2). However, post hoc analysis of the gamma frequency band showed that such differences could not be explained by specific regional differences (i.e., frontal) $[\mathrm{F}(1,107)=2.914, \mathrm{p}=0.091]$. The overall relative powers of other frequency bands did not differ between the two groups.

\section{DISCUSSION}

The findings of the present study suggest that the overall absolute powers could distinguish PTSD from anxiety disorders. Among specific frequency bands, the overall absolute delta powers were increased in patients with PTSD than those with anxiety disorders, and such differences were largely explained by the increased delta powers in the posterior regions. Additionally, the frontal absolute gamma powers and overall relative gamma powers were increased in PTSD compared to anxiety disorders. To our knowledge, this

Table 1. Demographic and clinical characteristics of study groups

\begin{tabular}{|c|c|c|c|c|c|c|c|}
\hline & \multicolumn{2}{|c|}{ PTSD $(\mathrm{N}=36)$} & \multicolumn{2}{|c|}{ Anxiety disorders $(\mathrm{N}=79)$} & \multicolumn{3}{|c|}{ Group comparison } \\
\hline & Mean & SD & Mean & SD & $\mathrm{t}$ & df & $\mathrm{p}$ \\
\hline \multirow[t]{2}{*}{ Age (years) } & 33.6 & 10.2 & 34.0 & 9.5 & -0.185 & 113 & 0.854 \\
\hline & $\mathrm{N}$ & $\%$ & $\mathrm{~N}$ & $\%$ & $\chi^{2}$ & $\mathrm{df}$ & $\mathrm{p}$ \\
\hline Gender & & & & & 2.017 & 1 & 0.166 \\
\hline Male & 14 & 38.9 & 42 & 53.2 & & & \\
\hline Female & 22 & 61.1 & 37 & 46.8 & & & \\
\hline Handedness & & & & & 1.347 & 2 & 0.510 \\
\hline Right & 33 & 91.7 & 73 & 92.4 & & & \\
\hline Left & 3 & 8.3 & 4 & 5.1 & & & \\
\hline Both & 0 & 0.0 & 2 & 2.5 & & & \\
\hline Being medicated & 31 & 86.1 & 64 & 81.0 & 0.447 & 1 & 0.602 \\
\hline
\end{tabular}

PTSD: posttraumatic stress disorder, SD: standard deviation

Table 2. Medication status of study groups

\begin{tabular}{|c|c|c|c|c|c|c|c|}
\hline \multirow{2}{*}{ Medication status } & \multicolumn{2}{|c|}{ PTSD $(\mathrm{N}=36)$} & \multicolumn{2}{|c|}{ Anxiety disorders $(\mathrm{N}=79)$} & \multicolumn{3}{|c|}{ Group comparison } \\
\hline & $\mathrm{N}$ & $\%$ & $\mathrm{~N}$ & $\%$ & $\chi^{2}$ & $\mathrm{df}$ & $\mathrm{p}$ \\
\hline Antipsychotic agent(s) & 16 & 44.4 & 7 & 8.9 & 19.571 & 1 & $<0.001$ \\
\hline Benzodiazepine(s) & 27 & 75.0 & 61 & 77.2 & 0.068 & 1 & 0.815 \\
\hline Antidepressant(s) & 25 & 69.4 & 61 & 77.2 & 0.792 & 1 & 0.488 \\
\hline Dose equivalents & Mean & SD & Mean & SD & $\mathrm{t}$ & df & $\mathrm{p}$ \\
\hline Olanzapine equivalent & 0.65 & 1.00 & 0.15 & 0.66 & 2.72 & 49.38 & 0.01 \\
\hline Lorazepam equivalent & 0.78 & 0.74 & 0.63 & 0.53 & 1.25 & 113 & 0.22 \\
\hline Imipramine equivalent & 6.07 & 17.51 & 10.52 & 21.55 & -1.16 & 0.25 & 0.25 \\
\hline
\end{tabular}

PTSD: posttraumatic stress disorder, SD: standard deviation 


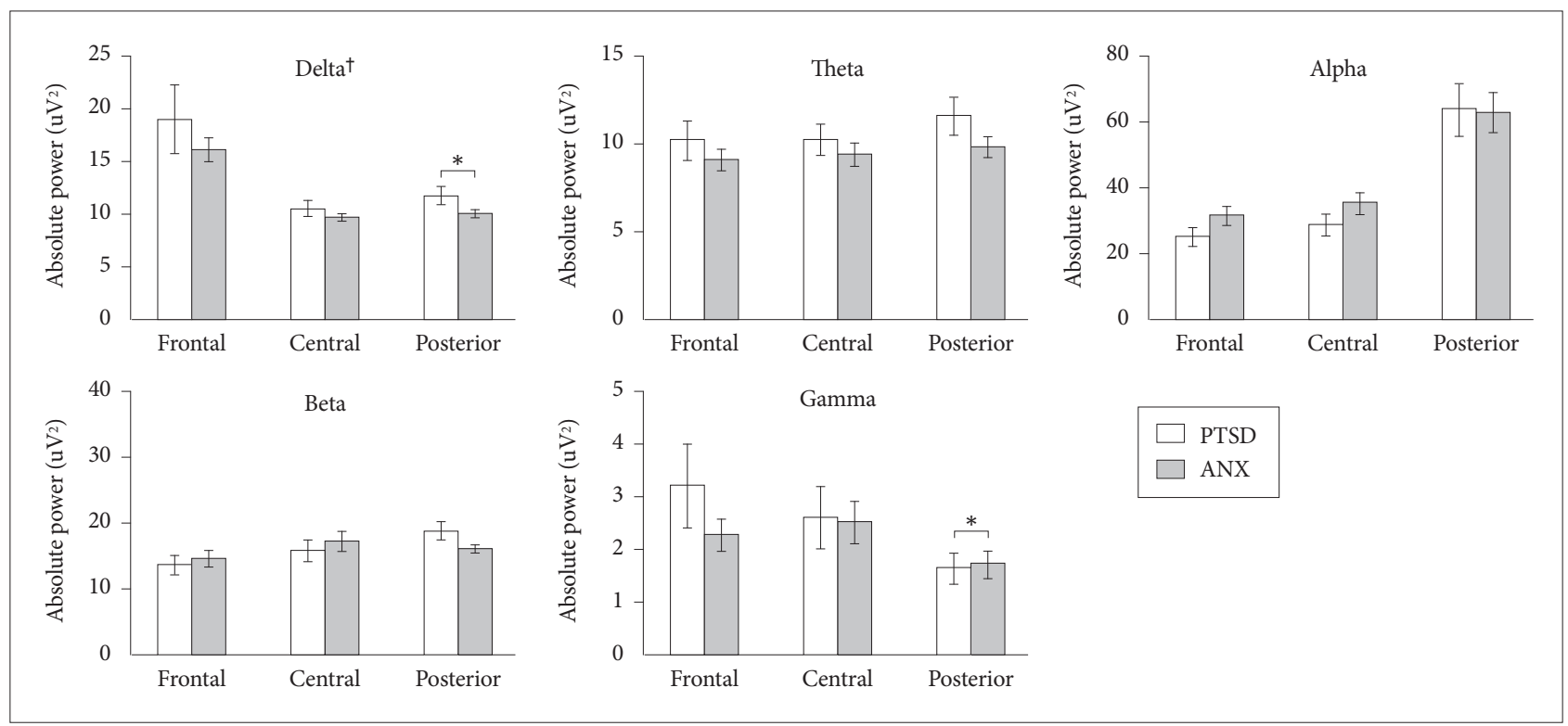

Figure 1. Absolute powers of the each frequency band under the resting-state eyes-closed condition. Horizontal bars show the standard error of the mean. 'overall absolute delta powers: posttraumatic stress disorder $(P T S D)>$ anxiety disorders $[F(1,331)=4.363, p=0.037]$, *post hoc analysis showed that the absolute delta powers of the posterior cerebral region were significantly increased in PTSD compared to anxiety disorders $[F(1,107)=4.001, p=0.048]$. An additional post hoc analysis was performed due to the possible group by region interaction effects in the gamma frequency band, which showed that the absolute gamma powers of the frontal region were increased in PTSD compared to anxiety disorders $[F(1,107)=4.138, p=0.044]$. PTSD: posttraumatic stress disorder, ANX: anxiety disorders.

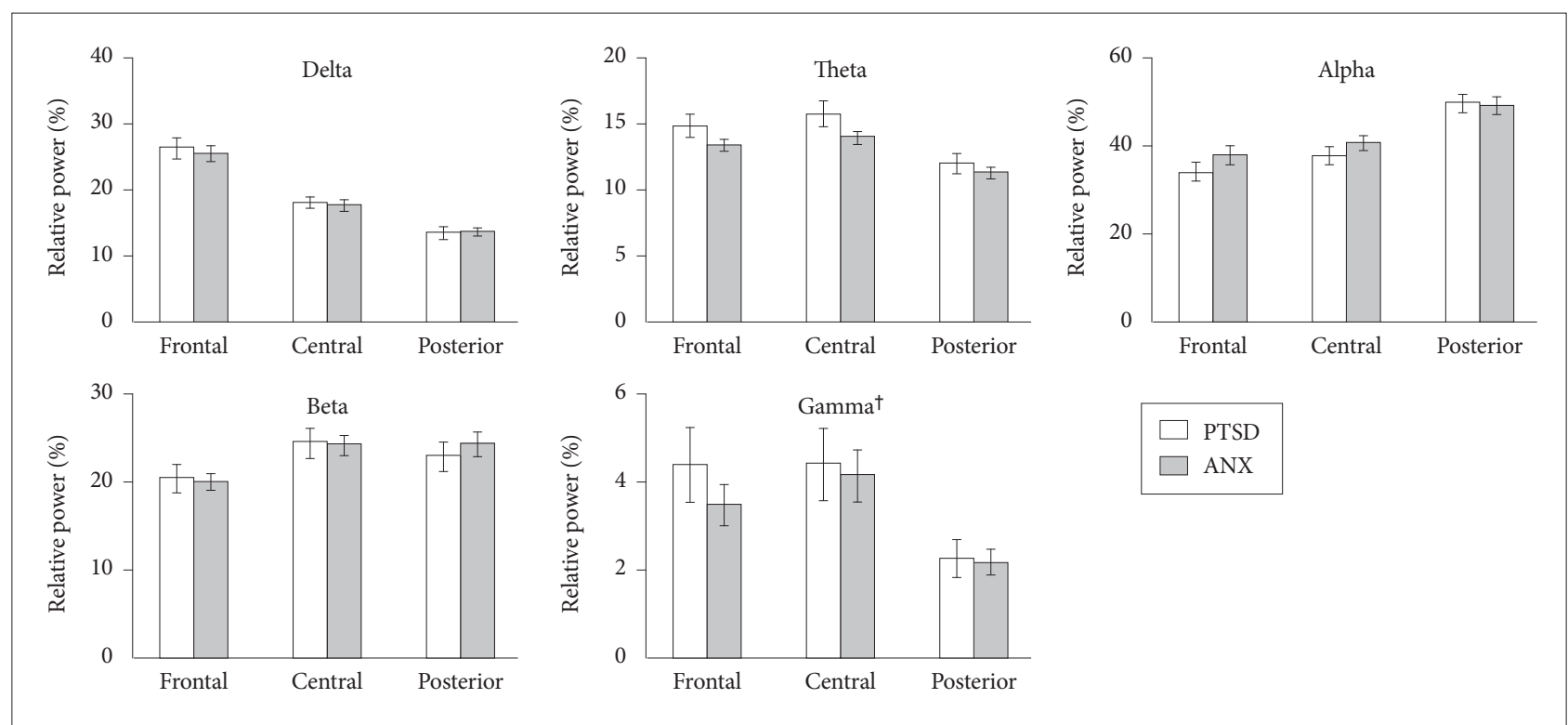

Figure 2. The relative powers of the each frequency band under the resting-state eyes-closed condition. Horizontal bars show standard error of the mean. 'overall relative gamma powers: posttraumatic stress disorder $(P T S D)>$ anxiety disorders $[F(1,331)=3.965, p=0.047]$. PTSD: posttraumatic stress disorder, ANX: anxiety disorders.

is the first study to directly compare resting-state qEEG findings of PTSD with anxiety disorders. Additionally, this is the first study to classify and quantify psychotropic medications and also to have controlled for them to allow for a more precise interpretation of resting-state qEEG findings.

Our results seem consistent with the up-to-date understanding of the neurobiology of PTSD which is hypothesized to be distinct from anxiety disorders. While both disorders share the phenomena of excessive fear, several other features are more characteristic of PTSD which includes negative alterations in mood and cognition, intrusive re-experiencing symptoms, and hypervigilance. ${ }^{33}$ Current study presented with results of increased frontal absolute gamma powers and overall relative gamma powers in PTSD compared to anxiety disorders. 
Gamma oscillations are hypothesized to reflect working memory load. ${ }^{34}$ Findings of attentional properties in PTSD is known to be disrupted in means of either failure of disengagement regarding emotional or fear stimuli with rapid reaction time, or avoidance of threat stimuli with slower reaction time. ${ }^{35}$ Moreover, cognitive alterations in PTSD are not only restricted to attentional problems, ${ }^{36}$ but also in the processing of trauma-unrelated emotional information and trauma-related information. ${ }^{37}$ In PTSD, activities of dorsal anterior cingulate cortex and hippocampus are found to be increased during cognitive processing, while activities are decreased in the same regions with increased activity in amygdala when trauma-related information is being processed. ${ }^{37}$ Regarding working memory, emotional working memory capacity is found to be decreased in PTSD, ${ }^{38}$ with functional connectivity studies showing the possibility of inefficient working memory systems in PTSD. ${ }^{39}$ Our study findings of increased frontal absolute gamma powers and overall relative gamma powers may reflect hypervigilance and, overactive and possibly inefficient mobilization of the central executive system in PTSD compared to anxiety disorders.

Our study also presented with findings of increased posterior delta powers in PTSD. Deactivation of visual cortex during color Stroop task and traumatic stimuli have been found in women with PTSD, ${ }^{40,41}$ and abnormal amplitude of low-frequency fluctuation (ALFF) signals in middle occipital gyrus were found in a resting-state functional magnetic resonance imaging study of PTSD patients. ${ }^{42}$ Along with previous study findings, our study result of increased posterior absolute delta powers might underlie deficits of visual processing area in PTSD, a finding that might distinguish PTSD from anxiety disorders. Meanwhile, previous studies have found that cumulative life stress loading is related to increased delta powers ${ }^{43}$ and another study's results showed that cumulative effect of life adversities were structurally related to volume reductions in the medial prefrontal cortex, anterior cingulate cortex, and the insular cortex. ${ }^{44}$ Although such previous study findings were not restricted to posterior brain regions or the occipital lobe, the relationship of life stress and delta powers, and adversity with brain regions that are related to PTSD may be one explanation of current study findings of increased delta powers of the posterior region in PTSD compared to anxiety disorders.

This study had several limitations. First, upon including patients with PTSD, we could not completely obtain and control for the history of closed head trauma. Although the probability of previous head trauma in PTSD patients in this study seems unlikely to be high, it is possible that the increased absolute delta powers may indicate a change toward slow waves after head trauma. Second, although we classified and quantified all the medications taken to control for their effects and also excluded patients who were taking doses more than two standard deviations from the mean for each class of medications, it is still possible that the EEG comparison data were not completely free from effects of medication. Both the symptoms of anxiety and the medications used to alleviate it can modify resting-state EEG findings, most common effects being increased beta frequency during anxiety states and diffuse slowing of resting EEG upon the use of benzodiazepines or antipsychotics. ${ }^{45,46}$ However, individuals with over-threshold symptoms implying the diagnosis of anxiety disorder or PTSD are likely to be treated in either in- or out-patient clinical setting, a setting in which they are likely to receive pharmacotherapy. Thus, considering the scarcity of qEEG studies of PTSD patients undergoing medication, it could be said that the current study has its implication upon the clinical interpretation of qEEG in a hospital setting.

In conclusion, overall absolute delta powers and overall relative gamma powers were increased in the PTSD group and differentiated them from anxiety disorder group. Absolute gamma powers of the frontal region were found to be increased in the PTSD group, which might underlie overactive and inefficient gathering of working memory systems in PTSD. Increased gamma powers may serve as a potential treatment target for symptoms of hypervigilance in PTSD with modalities of biofeedback or neuromodulation. Also, increased absolute delta powers of the posterior cerebral region compared to anxiety disorder group, may associate to visual deficits of PTSD. Since the defect in posterior cerebral regions seems to distinguish between PTSD and anxiety disorders, future replication studies are warranted if it could serve as a potential biomarker differentiating the two disorders. Furthermore, future replication studies could make use of machine learning technique to differentiate the two disorders with qEEG findings.

\section{Acknowledgments}

This research was supported by the Basic Science Research Program through the National Research Foundation of Korea (NRF) funded by the Ministry of Education (NRD-2017R1D1A1B03036385, http://www.nrf. re.kr) and Research Resettlement Fund for the new faculty of SNU.

\section{REFERENCES}

1. Careaga MBL, Girardi CEN, Suchecki D. Understanding posttraumatic stress disorder through fear conditioning, extinction and reconsolidation. Neurosci Biobehav Rev 2016;71:48-57.

2. Shin LM, Liberzon I. The neurocircuitry of fear, stress, and anxiety disorders. Neuropsychopharmacology 2010;35:169-191.

3. American Psychiatric Association. DSM-IV-TR: Diagnostic and Statistical Manual of Mental Disorders, Text Revision. Washington, DC; American Psychiatric Association; 2000.

4. American Psychiatric Association. Diagnostic and Statistical Manual of Mental Disorders (DSM- $5^{\circledR}$ ). Washington, DC: American Psychiatric Pub; 2013.

5. Etkin A, Wager TD. Functional neuroimaging of anxiety: a meta-anal- 
ysis of emotional processing in PTSD, social anxiety disorder, and specific phobia. Am J Psychiatry 2007;164:1476-1488.

6. Milad MR, Quirk GJ. Neurons in medial prefrontal cortex signal memory for fear extinction. Nature 2002;420:70-74.

7. Delgado MR, Nearing KI, LeDoux JE, Phelps EA. Neural circuitry underlying the regulation of conditioned fear and its relation to extinction. Neuron 2008;59:829-838.

8. Urry HL, Van Reekum CM, Johnstone T, Kalin NH, Thurow ME, Schaefer HS, et al. Amygdala and ventromedial prefrontal cortex are inversely coupled during regulation of negative affect and predict the diurnal pattern of cortisol secretion among older adults. J Neurosci 2006;26:4415-4425.

9. Wager TD, Davidson ML, Hughes BL, Lindquist MA, Ochsner KN. Prefrontal-subcortical pathways mediating successful emotion regulation. Neuron 2008;59:1037-1050.

10. Welborn BL, Papademetris X, Reis DL, Rajeevan N, Bloise SM, Gray JR. Variation in orbitofrontal cortex volume: relation to sex, emotion regulation and affect. Soc Cogn Affect Neurosci 2009;4:328-339.

11. Russo SJ, Murrough JW, Han MH, Charney DS, Nestler EJ. Neurobiology of resilience. Nat Neurosci 2012;15:1475-1484.

12. Davidson RJ. Affective style, psychopathology, and resilience: brain mechanisms and plasticity. Am Psychol 2000;55:1196-1214.

13. Shin LM, Wright CI, Cannistraro PA, Wedig MM, McMullin K, Martis $\mathrm{B}$, et al. A functional magnetic resonance imaging study of amygdala and medial prefrontal cortex responses to overtly presented fearful faces in posttraumatic stress disorder. Arch Gen Psychiatry 2005;62:273-281.

14. Kim MJ, Chey J, Chung A, Bae S, Khang H, Ham B, et al. Diminished rostral anterior cingulate activity in response to threat-related events in posttraumatic stress disorder. J Psychiatr Res 2008;42:268-277.

15. Admon R, Milad MR, Hendler T. A causal model of post-traumatic stress disorder: disentangling predisposed from acquired neural abnormalities. Trends Cogn Sci 2013;17:337-347.

16. Moscovitch DA, Santesso DL, Miskovic V, McCabe RE, Antony MM, Schmidt LA. Frontal EEG asymmetry and symptom response to cognitive behavioral therapy in patients with social anxiety disorder. Biol Psychol 2011;87:379-385.

17. Knott VJ, Bakish D, Lusk S, Barkely J Perugini M. Quantitative EEG correlates of panic disorder. Psychiatry Res 1996;68:31-39.

18. Sachs G, Anderer P, Dantendorfer K, Saletu B. EEG mapping in patients with social phobia. Psychiatry Res 2004;131:237-247.

19. Begić D, Hotujac L, Jokić-Begić N. Electroencephalographic comparison of veterans with combat-related post-traumatic stress disorder and healthy subjects. Int J Psychophysiol 2001;40:167-172.

20. Todder D, Levine J, Abujumah A, Mater M, Cohen H, Kaplan Z. The quantitative electroencephalogram and the low-resolution electrical tomographic analysis in posttraumatic stress disorder. Clin EEG Neurosci 2012;43:48-53.

21. Imperatori C, Farina B, Quintiliani MI, Onofri A, Gattinara PC, Lepore $\mathrm{M}$, et al. Aberrant EEG functional connectivity and EEG power spectra in resting state post-traumatic stress disorder: A sLORETA study. Biol Psychol 2014;102:10-17.

22. Jokić-Begić N, Begić D. Quantitative electroencephalogram (qEEG) in combat veterans with post-traumatic stress disorder (PTSD). Nord J Psychiatry 2003;57:351-355.

23. Lee S-H, Yoon S, Kim JI, Jin SH, Chung CK. Functional connectivity of resting state EEG and symptom severity in patients with post-traumatic stress disorder. Prog Neuropsychopharmacol Biol Psychiatry 2014;51:51-57.

24. Shim M, Im C, Lee S. Disrupted cortical brain network in post-traumatic stress disorder patients: a resting-state electroencephalographic study. Transl Psychiatry 2017;7:e1231.

25. Jang KI, Shim M, Lee SM, Huh HJ, Huh S, Joo JY, et al. Increased beta power in the bereaved families of the $S$ ewol ferry disaster: a paradoxical compensatory phenomenon? A two channel electroencephalography study. Psychiatry Clin Neurosci 2017;71:759-768.
26. World Health Organization. The ICD-10 Classification of Mental and Behavioural Disorders : Diagnostic Criteria for Research. Geneva; World Health Organization; 1993.

27. Gardner DM, Murphy AL, O’Donnell H, Centorrino F, Baldessarini RJ. International consensus study of antipsychotic dosing. Am J Psychiatry 2010;167:686-693.

28. Ashton CH. Benzodiazepines: How They Work and How to Withdraw. The Ashton Manual. Newcastle: Newcastle University; 2006.

29. Bollini P, Pampaliona S, Tibaldi G, Kupelnick B, Munizza C. Effectiveness of antidepressants: meta-analysis of dose-effect relationships in randomised clinical trials. Br J Psychiatry 1999;174:297-303.

30. Nierenberg AA, Greist JH, Mallinckrodt CH, Prakash A, Sambunaris A, Tollefson GD, et al. Duloxetine versus escitalopram and placebo in the treatment of patients with major depressive disorder: onset of antidepressant action, a non-inferiority study. Curr Med Res Opin 2007; 23:401-416.

31. Kim S, Shin JE, Kim MJ, Kwon JS, Choi SH. Correlation between Quantitative Electroencephalogram Findings and Neurocognitive Functions in Patients with Obsessive-Compulsive Disorder and Schizophrenia. Korean J Biol Psychiatry 2016;23:193-198.

32. Son K, Choi J, Lee J, Park S, Lim J, Lee J, et al. Neurophysiological features of Internet gaming disorder and alcohol use disorder: a restingstate EEG study. Transl Psychiatry 2015;5:e628.

33. Sheynin J, Liberzon I. Circuit dysregulation and circuit-based treatments in posttraumatic stress disorder. Neurosci Lett 2017;649:133-138.

34. Roux F, Uhlhaas PJ. Working memory and neural oscillations: alphagamma versus theta-gamma codes for distinct WM information? Trends Cogn Sci 2014;18:16-25.

35. Phaf RH, Kan KJ. The automaticity of emotional Stroop: A meta-analysis. J Behav Ther Exp Psychiatry 2007;38:184-199.

36. Armengol CG, Cavanaugh-Sawan A. Differential Performance of Post-traumatic stress disorder (PTSD), Attention deficit hyperactivity disorder (ADHD), and a Non-Clinical Control Group on Tests of Attention and Inhibition. Rev Esp Neurophysiol 2003;5:65-79.

37. Brown VM, Morey RA. Neural systems for cognitive and emotional processing in posttraumatic stress disorder. Front Psychol 2012;3:449.

38. Schweizer S, Dalgleish T. Emotional working memory capacity in posttraumatic stress disorder (PTSD). Behav Res Ther 2011;49:498-504.

39. Shaw ME, Moores KA, Clark RC, McFarlane AC, Strother SC, Bryant RA, et al. Functional connectivity reveals inefficient working memory systems in post-traumatic stress disorder. Psychiatry Res 2009;172: 35-241.

40. Bremner JD, Narayan M, Staib LH, Southwick SM, McGlashan T, Charney DS. Neural correlates of memories of childhood sexual abuse in women with and without posttraumatic stress disorder. Am J Psychiatry 1999;156:1787-1795.

41. Bremner JD, Vermetten E, Vythilingam M, Afzal N, Schmahl C, Elzinga B, et al. Neural correlates of the classic color and emotional stroop in women with abuse-related posttraumatic stress disorder. Biol Psychiatry 2004;55:612-620.

42. Yin Y, Li L, Jin C, Hu X, Duan L, Eyler LT, et al. Abnormal baseline brain activity in posttraumatic stress disorder: a resting-state functional magnetic resonance imaging study. Neurosci Lett 2011;498:185-189.

43. Marshall AC, Cooper NR. The association between high levels of cumulative life stress and aberrant resting state EEG dynamics in old age. Biol Psychol 2017;127:64-73.

44. Ansell EB, Rando K, Tuit K, Guarnaccia J, Sinha R. Cumulative adversity and smaller gray matter volume in medial prefrontal, anterior cingulate, and insula regions. Biol Psychiatry 2012;72:57-64.

45. Centorrino F, Price BH, Tuttle M, Bahk WM, Hennen J, Albert MJ, et al. EEG abnormalities during treatment with typical and atypical antipsychotics. Am J Psychiatry 2002;159:109-115.

46. Buchsbaum MS, Hazlett E, Sicotte N, Stein M, Wu J, Zetin M. Topographic EEG changes with benzodiazepine administration in generalized anxiety disorder. Biol Psychiatry 1985;20:837-842. 